Témoigner Témoigner. Entre histoire et mémoire

Getuigen Revue pluridisciplinaire de la Fondation Auschwitz

$123 \mid 2016$

Traduire le témoignage

\title{
Entretien avec Marion Pillé
}

Le Verfügbar aux enfers: interview met Marion Pillé

\section{Daniel Weyssow}

\section{(2) OpenEdition}

\section{Journals}

\section{Édition électronique}

URL : https://journals.openedition.org/temoigner/5084

DOI : 10.4000/temoigner.5084

ISSN : 2506-6390

\section{Éditeur :}

Éditions du Centre d'études et de documentation Mémoire d'Auschwitz, Éditions Kimé

\section{Édition imprimée}

Date de publication : 1 octobre 2016

Pagination : 27-33

ISBN : 987 2-9600926-4-6

ISSN : 2031-4183

Référence électronique

Daniel Weyssow, «Entretien avec Marion Pillé », Témoigner. Entre histoire et mémoire [En ligne], 123 |

2016, mis en ligne le 02 novembre 2021, consulté le 04 novembre 2021. URL : http://

journals.openedition.org/temoigner/5084; DOI : https://doi.org/10.4000/temoigner.5084 


\section{ENTRETIEN AVEC MARION PILLÉ}

MUSIQUE L'opérette de Germaine Tillion rédigée au camp de concentration de Ravensbrück sera interprétée du 29 novembre au 10 décembre 2016 au Théâtre Marni à Bruxelles. Nous avons rencontré la metteure en scène, Marion Pillé, deux mois après l'étape de travail présentée par sa compagnie Les

Souffleuses de Chaos, le 11 novembre 2015, à la Maison de la Création. L'invitation portait en exergue la présentation suivante: "Sur la piste jonchée de cheveux tondus d'un cirque macabre à l'humour grinçant, quatre comédiennes chantent et dansent pour défier la mort et la barbarie.»

$\rightarrow$ Entretien mené par Daniel Weyssow

le mardi 19 janvier 2016 à la Fondation Auschwitz
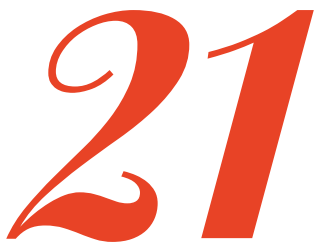

octobre 1943, Germaine Tillion est déportée à Ravensbrück pour acte de résistance. En octobre 1944, grâce à la solidarité de ses camarades et bien que l'acte d'écriture soit puni de la peine de mort, elle se livre à un véritable tour de force : écrire. En effet, persuadée que l'humour et la compréhension de leur univers sont les derniers remparts contre la déshumanisation, elle décrit, dans une opérette sans concession, les conditions de vie des déportées : Le Verfügbar aux Enfers.

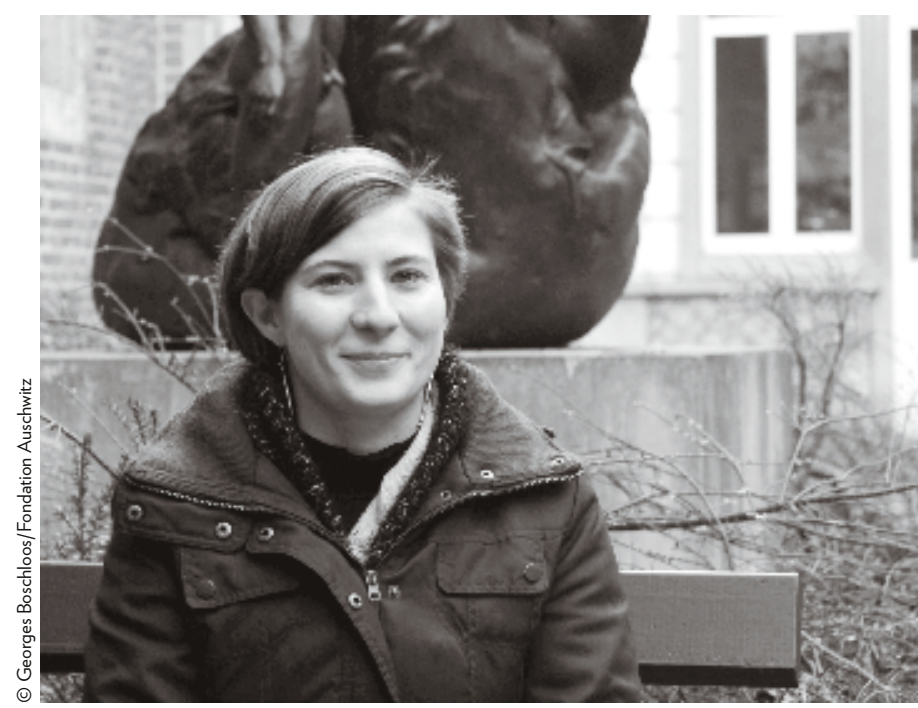

Vous avez été formée principalement dans deux institutions, en France et en Belgique, l'école Florent à Paris, et l'INSAS à Bruxelles, d'où vous êtes sortie en 2012. On peut avancer que pour réaliser le Verfügbar aux Enfers. Une Opérette à Ravensbrück de Germaine Tillion, il était justement nécessaire d'avoir un solide bagage pour aborder cette pièce qui s'avère subtile et compliquée à mettre en scène et à interpréter. Est-ce la première création de votre compagnie?

Marion Pillé : Non, c'est la deuxième création de la compagnie. Mais cela faisait déjà très longtemps que je voulais travailler sur le Verfügbar aux Enfers. C'était d'ailleurs mon projet de fin d'études à l'INSAS, mais au vu des réactions au travail déjà engagé,j'avais besoin de passer d'abord par des formes plus légères, c'est-à-dire par un autre projet.

Vos professeurs estimaient-ils prématuré de vous lancer dans un tel projet de fin d'études?

Des professeurs m’ont dit que je ne pourrais porterce projet, tel que je le pensais, avant d'avoir quarante ans... $\bullet \bullet$ 
Quels avis avaient-ils sur la question?

J'ai préparé et présenté à l'INSAS une étape de trente minutes, qui correspondait quasiment à l'acte On avait fait quelquescoupes pour avoir quelque chose de cohrient. Hy áleaures noupd ment sur la nsions, d'abord cr la problématique des camps de continence tion aujourd'hui Déjà à l'époque (en 2012) cela me tion aujourd hui. Dejà à lépoque (en 2012) cela me enblat pourtant nécessaire. Je trouvais indispentenu de l'a co question du nazisme. Compte tenu de lactualité, on sent à présent que les gen comprent nienx quin aut en parler, alors qu'il y a quatre ans, beá oup de gens me disaient «mais haine de lautre."

Cela concernait en fait la réception du sujet et pas la pièce elle-même ?

Il y a d'abord le sujet, puis la forme. Beaucoup de gens m'ont demandé pourquoi je voulais monter un texte que lauteure navait laisse publier que très tarune forme de trahisonune forme de trahison.

Dès le moment où Germaine Tillion a livré son texte lopérette a été représentée..

Oui. Et Germaine Tillion était présente à sa création, en juin 2007, au Théâtre du Châtelet, elle l’a vue

Donc elle était d'accord que l'on monte le spectacle ?

Absolument... Je ne l'aurais pas fait si elle avait refusé. La question me semble-t-il se poserait différemment.

Pour quelle raison cette opérette était-elle restée dans ses tiroirs?

Une des raisons qưelle donne de ne pas avoir publié le Verfügbar tient dans sa crainte que le public ne comprenne pas son recours à l'humour. Elle craignait que dans les cal dans les camps si celles-ci pouvaient être racontées en humour et en chansons. Ce qui a dû jouer aussi, C'est son groupe d'amies survivantes de Ravensbrück qui l'a convaincue en arguant que ce texte parvenait à transmettre une vérité de ce qu’elles avaient vécues.

Ce qui est paradoxal dans cette œuvre, ce qui la traverse et qui complique un peu les choses, c'est qu'il ne
s'agit pas d'une œuvre écrite pour être montrée hors du sagit pas dune œun écrite pour être montrée hors du contexte du camp. Kle était destinée, en y développan un analyse du fonction d'

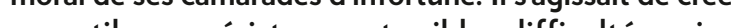
prén pour resister aux tentibles difficultés qui se presenta du camp lose pre contexte du camp pose probleme puisquili a été conçu pour un usage interne Cest donc complique. Comm lescomedies occuper la place des déportées dont il est question dan la pièce ? Comment operrer le transfert de la parole des qu'à elles-mêmes?

C'est vrai, une des difficultés relève du fait, et on le sent dans le travail, que le public auquel on s'adresse n'est pas du tout le public auquel le texte est destiné. qui save qui sulté consiste à recréer cette commuestion. La difficulté consiste à recreerer cette communauté d'écoute puisqu'il s'agit d'un texte joué par des déportées pour des déportées. Elles ne lont en fait jamais joué, elle lont hu, nais il esteffective entendu par le même groupe de personnes. Comment arriver, aujourd'hui, à recréer ce sentiment d'appartenance à un même groupe ? La première difficulté pour les comédiennes a été de se demander comment interpréter des déportées, mais je n’ai justement pas voulu qu'elles jouent des déportées. Du coup, je me suis dit quau travers des marionnettes, il y avait moyen diobtenir cette matérialisation tout en assumant complètement qu'elles ne sont que les témoin de Charolte Delo été écrite. Ensuite, ily a les texte de Charlotte Delbo. Je trouve qu'elle decrit très bien son rapport à son «double dAuschwitz» comme elle l'appelle. AAuschwitz, ellen avait pas l'impression que cétait elle. Elle ne pouvait pas se reconnaitre dans ce corps décharné. Et lorsqu'elle est rentrée, elle n’apas pu s'imaginer ailleurs que dans le camp. Elle ne comprenait pas que les gens ne voient pas qu'elle était presque être. double érit queelle vit en permanence «dans un être double», avec son double déporté à côté d'elle qui parfois prend toute la place. Je trouvais aussi qu'

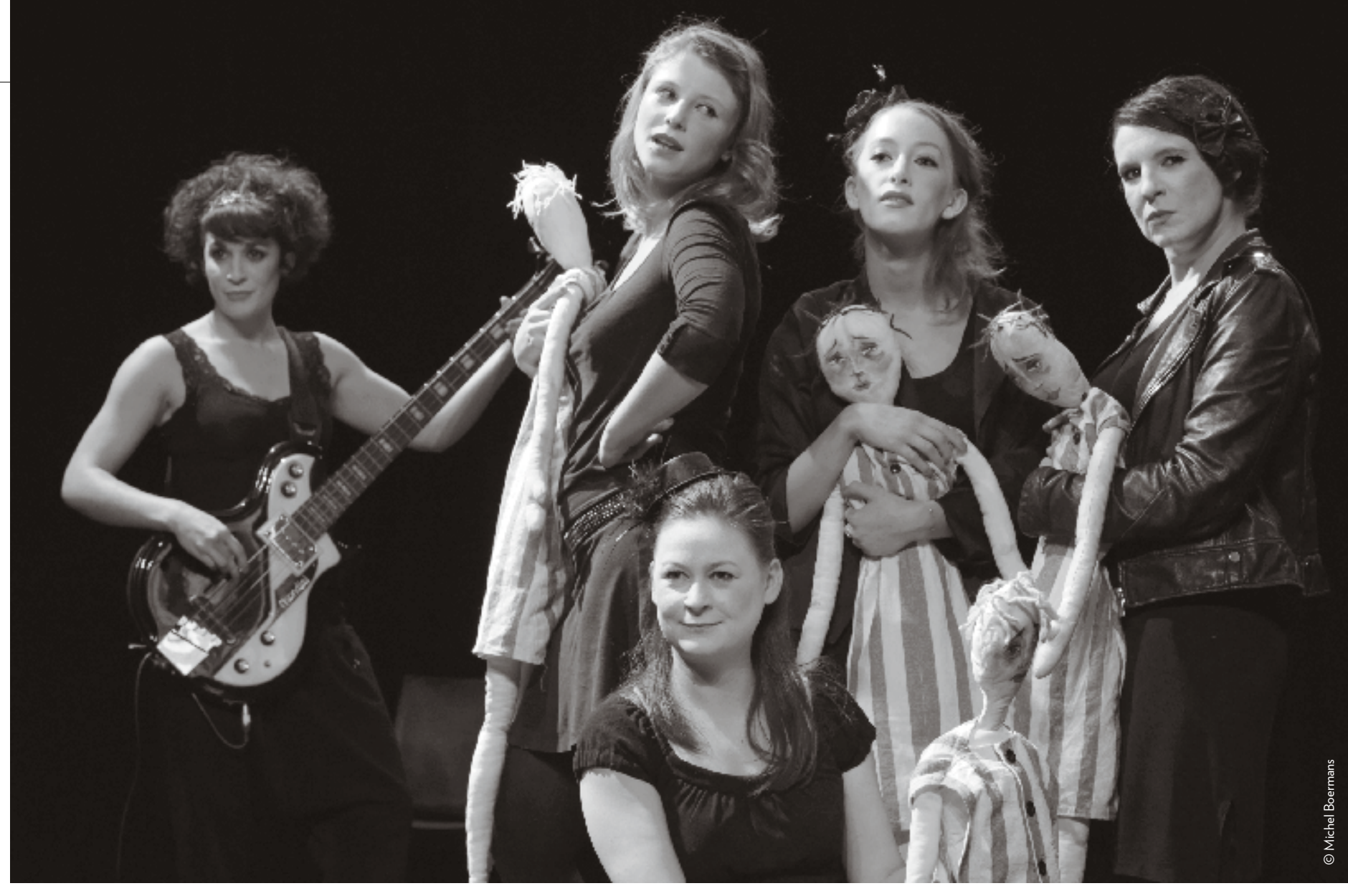

traumatique empêchait quelquefois les déportés de vivre le quotidien. Une des contraintes dans le jeu des comédiennes consiste donc à faire usage de ces poupees-marionnettes qui font 1 in2. Les comédiennesles auront toujours sur elles, seront obligées de les aime et de les detester en mêne temps parce quelles von prendre trop de place, voire acctam la place. Elles seront toujours là, même si lobjet est fait, en soi, pour être déposé, elles ne pourront jamais déposer

Comment en êtes-vous arrivée à vous intéresser l'œuvre de Germaine Tillion et au Verfügbar aux Enfers? l'œuvre de Germaine Tillion et au Verfügbar aux Enfers?
Comment avez-vous découvert ce texte et comment vous en êtes-vous emparé?

Au tout début, je comptais écrire l'histoire de mon grand-père, mais c'était compliqué à rapporter sur
scène et à confier à des comédiens. Il y avait un lien scène et à confier à des comédiens. Il y avait un lien du théâtre. Le texte de Germaine Tillion avait suffi- samment de points communs avec l'histoire de mon grand-père pour que je puisse y retrouver ce que je que je puisse le porter à la scèn... été déporté à Buchenwald à 17 ans. Il grand-pere

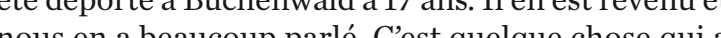
prous en a beaucoup parké. Cest quelque chose qui a profondément marqué mon enfance et mon adolescence. Il a essayé de nous transmettre des valeurs, de nous eduquer dansle respect delautre, de la difference, sociétés.

Était-il engagé dans la Résistance?

Oui, il s’est engagé dans le réseau de résistance de 'Abbé Blanc à Marseille. Il a été arrêté en août 1943 par la Gestapo.

Germaine Tillion a aussi été arrêtée pour faits de résistance, pas à Marseille, mais à Paris. Elle avait déjà ... d'exclusion et de haine pouvant voir le joute form 
•. vécu une expérience très originale et intéressante dans le cadre de la rédaction de sa thèse de doctorat que dirigeai Marcel Mauss, l' 'inventeur de l'ethnologie. II l'a envoyée en Algérie, dans les montagnes de l'Adour, pour y analyser mode de vie d'une communté berbère. Elle a décryp é puis décrit les rapports existants entre les membres de cette communaute. Sent-on, au travers du Verfugbar, une êne méthodologle appliquée au décryptage du mode de fonctionnement et de la finalité du camp, que elle perçu sous langle d un rapport
sur le travail, la mort et l'argent?

Oui, on sent que ce texte repose sur sa condition de scientifique et d'analyste déterminée à objective de scientifque et d'analyste déterminée à objectiver cette experience. Elle a observé les humains vivre en groupe. Le personnage principal de lacte 1 s'intitule découvre une nouvelle espèce, le Verfügb decouvre un. qu XIXe se au XIX siècle, y compris des humains dálleurs, pour endécrire toutes les caractéristiques physiquesdevant une assenble. On sent qu'elle a une viaie formation cientifique dethnologue. Par ailleurs, des ressorts comiquesnous font prendreconscience de toute lhorreur de leur condition. Ce naturaliste ne met aucun ion Verfügbar est maigre tient à hyper bizarre, reste debout 17 heures par jour..

On ne trouve pas dans les textes d'autres rescapés lironie pleine de verve de Germaine Tillion, destinée à lironie pleine de verve de Germaine Tillion, destinée à faire rire en pourfendant verbalement et en musique systeme concentrationnare. II ne semble pas que l'on puisse trouver beaucoup dautres exemples de cetype de rêsistance, sauffeut-être à Theresienstadt, si on pense a lopéra de Viktor Ullmann, "LEmpereur dAtlantis ou la Refus de la mort, ou la mort abdique. Ou encore a la pièce dHanus Hachenburg, "On a besoin d un fanhe n, qui a ter reurbe traduite récemment parla chercheuse Claire Audhuy. Cette pièce évoque également avec humour et esprit frondeur letat de dictature égnant a Theresientad. Pensez-vous quill soit possible détablir un parallele, en termes de résistance, avec les artistes de ce ghetto? Au mom où elle écrit sa pièce, Germaine Tillion est encore en pleine forme. Elle estime le temps de survie des deports a Ravensbrück à deux quà Auschwitz où l'espérance de survie était, pour les Juifs, de trois mois.

Elle parle assez bien des conditions d'écriture du Eüübar dans son étude intitulée «Ravensbrück». Verfugbar dans son étude intitulée «Ravensbrück». l'écrire à un mont où unes. Elle précise quelle a pu técire à un moment où un certain nombre de condicons étaient réunies. Elle et son connando étaient lices ces dé du prendre à leur charge sa pas on cele puis quelle puisse secacher pour écrire. Cette dénarche de ǵtéipe est en soi très particuliere. Sans cela illui aurait été impossible decrire. Elle dit que ça n'aurait plus été possible au-delà de ces trois semaines. Elle était trop plation les transports et les sélections devenaient plus frequents, une chambre à gaz avait été construite à Ravensbrück même, le danger devenait trop grand.

Est-ce que le fac-simile publié1 est bien le carnet original ? Car la précision de son écriture y est tellement reme Car person encto

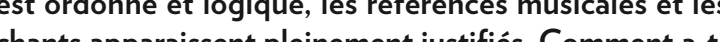
hats aparaissent pleinement justifies. Comment a-t a le pu fare pour développer une histore somme toute assez complexe dans un laps de temps si court et d' une

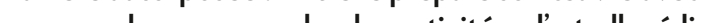
sule dans son coin?

Certaines chansons ont été écrites de façon collective, chacune des déportées du groupe devait troupas très bien, elle ne s'est pas beaucoup explique à pas très bien, elle ne s'est pas beaucoup expliquée à dit aut. lit aussi dans "Ravensbrick»", ce texte avait un but, es aider a survivre. Cetait pour Germaine Tillion une descondirions ilsvounentaice pour pouvoir lutter contre ça.

Le texte du Verfügbar est truffé de mots de vocabulaire qui ne peuvent être compris que par des déportés, voire uni peuvent être compris que par des déporäs, puisqu'il s'agit d'une discussion interne à propos du camp.

(1) Germaine Tillion, Le Verfügbar aux Enfers. Une opérette à Ravensbrück,
La Martinere, Paris, 2005. (2) Germaine Tillion, Une opérette à Ravensbrück, Points, Paris, 2007.
Des termes sont nés de traductions adaptées de mots allemands et le langage adopte nombre de formulations humoristiques, à l'exemple des tricoteuses et des cartes roses $^{3}$, le Innendienst, c'est-à-dire le service intérieu pour celles qui, épuisées, recevaient l'autorisation de passer la journée dans leur bloc ; l'Arbeitseinsatz transormé en Arbeiterszatz pour évoquer un pseudo burea dembauchage du camp ; Aspirine plutôt qu'Aufzehrin, la gardienne ; la Bekleidung, lentrepôt de vêtements ; Blockova, unot d argottchèque remplaçant Blockătest la doyenne du bloc; Blok 32 ; Bounekère (pour Bunker). Le texte, truffe didiomes, peut-il être compris sans une connaissance prélable de ce vocabulaire? Commen envisagez-vous cette difficulte? Comment le spectateur

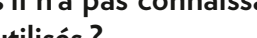

L’une des clés aura déjà été de comprendre le texte nous-mêmes. Les premiers temps de répétitions, on consacré les matinées à travailler rien que sur cetter quériode, sur la montée du nazisme en Allorique sur en Europe sur la répression des populations océ te sur la déportation dans toutes ses modilís. On et sur la déportation dans toutes ses modalités. On a a essayé de décoder le parcours type d'un dépoé on estarivé à la conclusion que c'était importé, et S'il y a de grandes bases, les expériences sont Silu a de les lisant tealement en lisant beaucoup. I fallait un bagage commun, des de répétition commencent par la lecture de témce de res: Jorge Sempun Robert Ant lue, Prino Levi, Charlotte Delbo...

Peut-être qu'initier une projection composée d'un

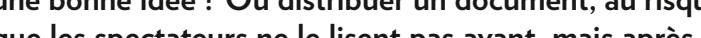
spectacle donc trop tard?

Oui, c'est ça, sans doute faudrait-il distribue l'information. Peut-être en présentant un lexique des

(3) Les tricoteuses sont les femmes qui étaient affiliées aux colonnes de travail den nuit les cartes roses sont les malades qui avaient obtenu au
Revier une dispense de travail. Ces deux groupes de femmes occupaient
les blocs pendant la journée (M.P.). mots dont le public aura besoin. Nous comptons orgaiser des rencontres avec le public. Je me suis rend compte, lors du débat après la présentation d'étape en novembre 2015, que les gens ressentent une espèce ur la facon aut ce texte peut amene à faire regé cur la facon dont ce texte peut an cette période peut nous faire réféchir àce aujourd'hui pont mémoriel m'intére qui pas' est important de continuer à parler de lápó mais je trouve qu'il faut surtout apprendre dation, mais je trouve qu'il faut surtout apprendre du passé pour eviter la rétération devénements semblables. pour éviter le retour de tels événements.

L'ironie qui opère dans l'opérette de Germaine Tillion thé à un tel niven quil semble peu appropié de parler d' " humour ", la pièce étant pleinement tragique.

Je ne trouve pas que ce soit une pièce comique, c'est el'intelligence, des pieds de nez au nazisme, aux nazi En tout cas, je ne fais pas cette pièce pour faire rire les poétique et artistique permet de touchise en forme poétique et artistique permet de toucher les spectaPeut-être que l'humour, la musique, la marionnette. ent-être que lhône de poésie, peuvent perionnette, pas d'être à la hauteur de la réalité, mais d'en saisi quelque chose Comment Germaine Tillion trouve-tquelque chose. Comment Germaine Tillion trouve-ttions d'avoir des mots sarcastiques fà à bar plus brutale? Elle dità cetégard que si elle a reco l'humour cest pere que 'humour c'est parce que c'est la meilleure arme. Elle autres unirover . ja

\section{Comment avez-vous trouvé le « ton » de la pièce, son}

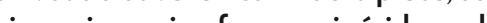
dit, dans son ế cet univers hallucinant ?

On a déjà dégagé quelques pistes, mais on est encore en train de chercher. Ce qui importe, c'est la juste distance entre les comédiennes et leurs personnages La question qui sest posée est de savoir comment interpreter des déportés. Je n'ai pas voulu qu'elles les porteuses de paroles des témoins. On a beaucoup 
-. travaillé cette question pour finalement décider de ne pas respecter la distribution de Germaine Tillion Nous avons recéc ensenble quáre personnages cor-

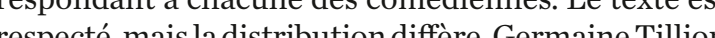
especté, mais la distribution dif́e. Germaine Tillion propose une dizaine de déportées, qui correspondent im cour d'une masse indifférenciée, où l'on ne distingue d'individu à des figures qui progressivement s'individulisent. Le pour fit par reconnitre des figures ligures ens person

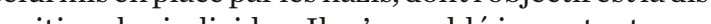
parition des individs. Harta diver à cette iǹ dienne travalle à partir de ce que lon a appelé entre nousleur

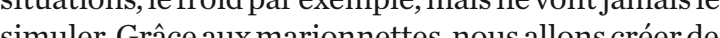
tableaux muets qui là vont imager..

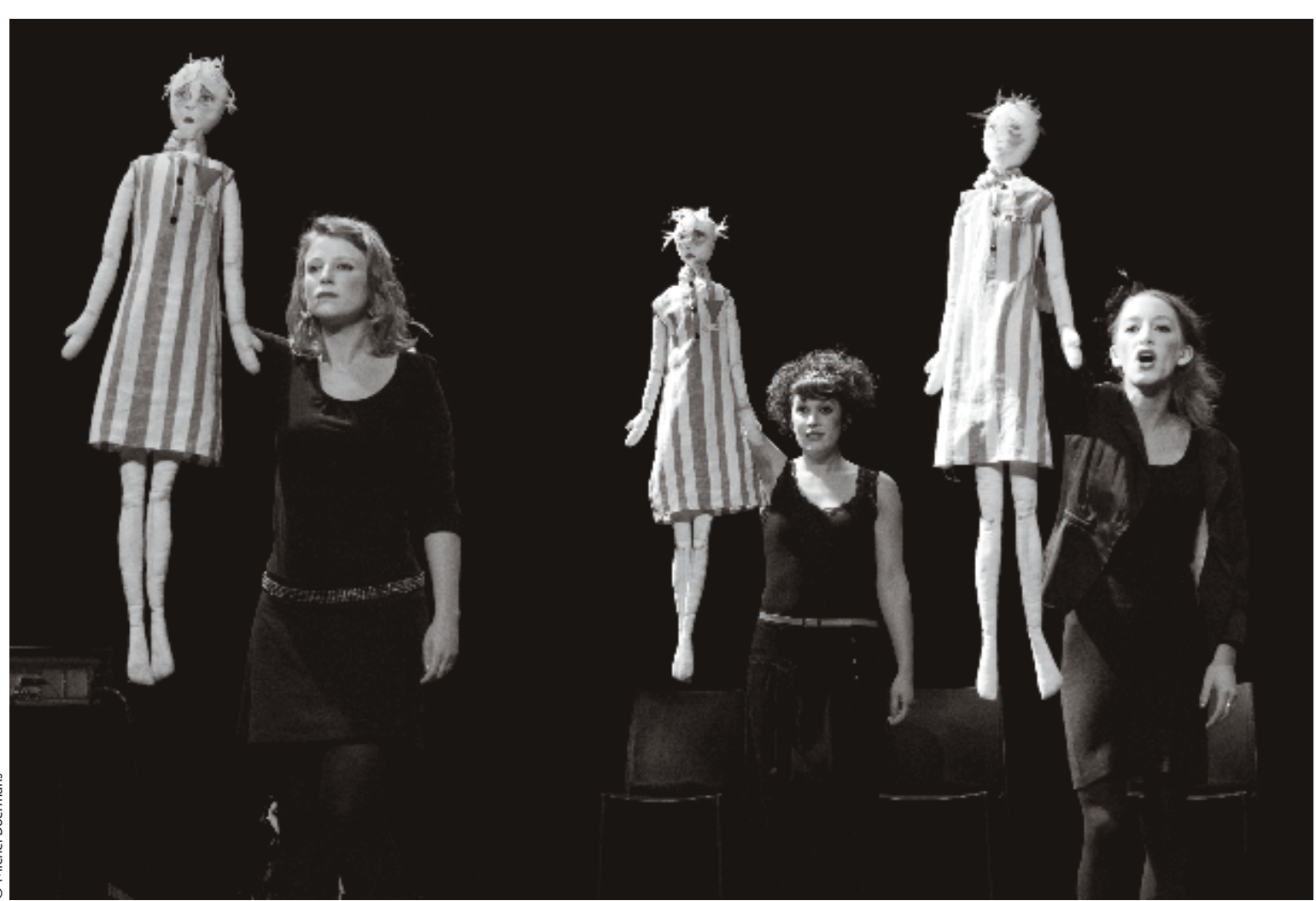

La question de l'indicibilité de l'expérience concentrionnaire a été longuement étudiée. S'il est en effet difficile de tout rapporter des expériences humaines, les déportés - et les romanciers - ont tout de même trouve des mots et des formules pour exprimer certains non-dits.

J'ai besoin qu'on sente sur le plateau que ce n'est comédiennes C'estun pariquipeutêtre risqú Vill utiliser d'autres arts que celui pour lequ. pétente. J'ai envie que les choses soient fagiles

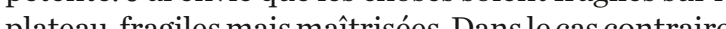
il me semble que quelque chos. Dansle cas contraire, rapport au propos.

Quand les comédiennes jouent de la guitare élecQique, elles partagent le même monde que celui des pectateurs, et non pas de facto, celui des camps. Mais pas une parole évidente. Parmi l'équipe, aucune n'est marion

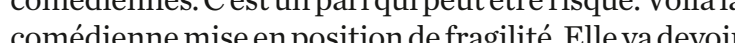

encore une fois, sans préparation, comment les spectaeurs pouraient-ils comprendre la logique du mécanisme mis en place?

L'idée pour la scénographie - qui était dans l'étape rès dénudée - n'est pas de reproduire un camp de concentration, mais dévoquer la réalité sans pour par endroits, mais on n'y retourne jamais vraime par endroits, mais on n'y retourne jamais vraiment. l'écriture de Germaine Tillion. On consta là dans lecriture de Germaine Tillion. On constate dans les rouvents de scène comme un emballement: elles relent, elles stimulent leur inagiraire par revocation

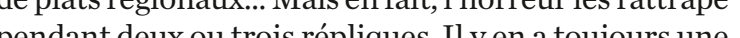
pendant deux ou trois répliques. Hy en a toujours une pour revenir a des réalités tres dures dont les autres personnages refusent de parler. Il est interressant de

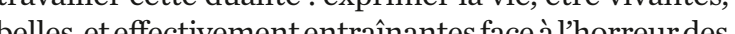

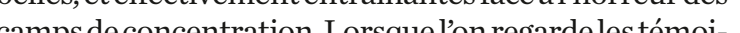
campsdeconcentration. Lorsquelon regarde lestémoignages des déportés, onles voit vivre.J Ja tétérappéepar elles ont ce plaisir-là aussi. J'avais enviek. en fait Ce plaisirla

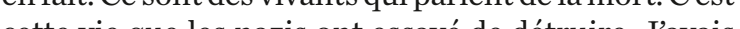
cette vie que les nazis ont essaye de détruire. Javais

\section{Germaine Tillion a égalementbien remplison rôle de}

mpli son rôle de personne ressource en parodiant l'opérette dOffenbach Orphée aux Enfers, elle-même une parodie d' Orphée et Eurydice de Gluck. Elle in erpretait au fond le role dOrpheee en espérant ramener de lenfer - Ravensbríck - ses compagnes derter qui, comma préterez l'air d' "Orphée aux Enfers »?

On l'entend, oui. Il y a un moment où elle s'en inspire, au tout début de l'acte 3 . On sent d'ailleurs fatigue et la détérioration de son état physique dan l'ecriture. Le premier acte est très rebondissant, avec des chansons tres entrainantes. Il est beaucoup plus nourri et long que les deux autres. Petit à petit cela endroits. On unt pusiqu pla trajet de Germaine Tillion dans son écriture.

D'accord, nous arrivons tout doucement au bout de cette interview. Quel message pensez-vous transmettre cette interview. Quel
au spectateur? (rires)
Face à la barbarie, la solution ne peut pas être de ermer les yeux ou de collaborer, ce qui est encore pire. te battre, com battre, comme Germaine Tillion, jusqu'au bout, aprè dis que silon veut que de tels actes se gén devienne dès mant, soyons fous, la nor à il faut conn d'exclusion et de haine. It fa dire non aux logiques laxclusion et de haine. Il faut creser un état de vigiforme d'éthe être citoyen tout sim. forme deethique, être citoyen tout simplement. II y

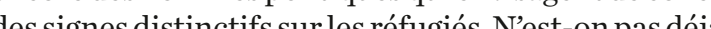
pes pas ? Con passé par là ? Comment peut-on à ce point oublier le de rester vistoire On a le devori, comme citoyen, prendre à reconnaître ces signes de dérives.

Oui, il faut apposer à ce qui se déroule maintenant les exemples du passé, être vigilant et que chacun réfléchisse ux conséquences des décisions qui sont prises. Germain Tillion a dit aussi qu'il fallait investir dans linstruction, ans l'enseignement. Est-ce que vous comptez attire également un public scolaire?

Le spectacle est monté pour tout public, avec la volonté de faire venir les écoles et les jeunes, qui son éfle ravenir. Jevoudrais engager un travail de Une série d'ateliers sera Une classes. Avant effet il est un peu dur de les lancer dans ce ger. En pièce s'ils n'ont pas un minimum de cons ce genre de piece sils non pas un. liers de théâtre liers de theâtre seront axés sur les liens qu'ils peuven aire avec leur quotidien ou le monde dans lequel on évolue.

Plus d'infos Texte écrit en déportation par Germaine Tillion
Une production : Les Souffleuses de Chaos. Mise en scène de Marion Pillé. Interprétation: Aurore Lacrosse, Sophie Maréchal, Marion $\widehat{A}$ Représentations prévues au Théêtre Marni à Bruxelles du
29 novembre au 10 décembre 2016. 